
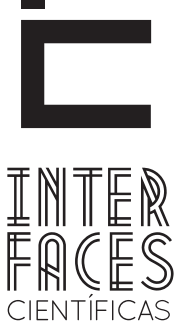

SAÚDE E AMBIENTE

ISSN IMPRESSO 2316-3313

E - ISSN 2316-3798

DOI - 10.17564/2316-3798.2017v5n3p37-46

\title{
ESTRESSE E ATIVIDADE FÍSICA EM MOTORISTAS DE ÔNIBUS URBANO EM UMA CAPITAL DO NORDESTE DO BRASIL
}

STRESS AND PHYSICAL ACTIVITY IN URBAN BUS DRIVERS IN A NORTHEAST CAPITAL OF BRAZIL ESTRÉS Y ACTIVIDAD FÍSICA EN LOS CONDUCTORES DE AUTOBÚS URBANO EN CAPITAL DEL NORESTE DE BRASIL

Rosa Luciana Prado ${ }^{1}$

Maria Hozana Santos Silva²

Marlizete Maldonado Vargas ${ }^{3}$

\section{RESUMO}

O estresse ocupacional pode contribuir de forma negativa na saúde do trabalhador. 0 objetivo deste trabalho foi identificar estresse, estressores e fatores de riscos em motoristas de ônibus urbano de Aracaju, SE. Pesquisa caracterizada como descritiva transversal com delineamento correlacional, utilizando os seguintes instrumentos de coleta: Inventário de Sintomas de Estresse de Lipp (ISSL), Questionário Internacional de Atividade Física (IPAQ - 8.0) e levantamento sociodemográficos. A amostra foi composta por 322 motoristas seleciona- dos de forma representativa e que voluntariamente se dispuseram a participar da pesquisa. Todos os sujeitos do estudo são do sexo masculino (média de idade de $37,16 \pm 8,79$ anos), $62,11 \%$ são casados, $27,02 \%$ solteiros, 5,59\% divorciados, 5,28\% declararam ter outro tipo de situação conjugal e $81,99 \%$ têm filhos. Entre os entrevistados, 46,89\% dos sujeitos apresentavam estresse e $46,18 \%$ relatou 0 tipo de organização do trabalho como fator estressor. Dos que apresentaram algum grau de estresse, $3,97 \%$ encontravam-se na fase de alerta, $88,74 \%$ 
na fase de resistência, 1,99\% na quase-exaustão e $5,29 \%$ na exaustão. Os fatores de riscos relacionados ao estresse foram: carga horária acima de 8 horas/dia; assaltos durante o trabalho; sedentarismo; IMC acima de $25 \mathrm{~kg} / \mathrm{m}^{2}$ e menos de 6 horas de sono diárias. Os resultados apontam ainda que $77,02 \%$ dos sujeitos da pesquisa apresentam baixos níveis de atividade física. Foi observada correlação negativa e significativa entre o nível de atividade física

\section{ABSTRACT}

Occupational stress can contribute negatively to worker health. The objective of this study was to identify stress, stressors and risk factors in urban bus drivers from Aracaju, SE. A cross - sectional descriptive study with a correlational design, using the following collection instruments: Lipp Stress Symptom Inventory (ISSL), International Physical Activity Questionnaire (IPAQ - 8.0) and socio - demographic survey. The sample consisted of 322 drivers who were representatively selected and willing to participate in the survey. All subjects were males (mean age $37.16 \pm 8.79$ years), $62.11 \%$ married, $27.02 \%$ single, $5.59 \%$ divorced, $5.28 \%$ reported having another type of marital status and $81.99 \%$ have children. Among the interviewees, $46.89 \%$ of the subjects presented stress and $46.18 \%$ reported the type of work organization as a stressor. Of those who presented some degree of stress, $3.97 \%$ were in the alert phase, $88.74 \%$ in the resistance e o de estresse $(r=-0,238 ; p<0,001)$. A presença de estresse, na fase de resistência, sugere que os motoristas têm desenvolvido resiliência aos estressores estudados.

\section{PALAVRAS CHAVE}

Esgotamento profissional, Estilo de vida sedentário, Motoristas de ônibus

phase, $1.99 \%$ in the near-exhaustion, and $5.29 \%$ in the exhaustion phase. The risk factors related to stress were: working day over 8 hours; Assaults during work; sedentary lifestyle; BMI above $25 \mathrm{~kg} / \mathrm{m}^{2}$ and less than 6 hours of sleep per day. The results also indicate that $77.02 \%$ of the subjects present low levels of physical activity. A negative and significant correlation was observed between physical activity level and stress level $(r=-0.238$, $p<0.001$ ). The presence of stress, in the resistance phase, suggests that drivers have developed resilience to the stressors studied.

\section{KEYWORDS}

Professional Exhaustion, Sedentary Lifestyle, Bus Drivers

\section{RESUMEN}

El estrés laboral puede contribuir negativamente en la salud de los trabajadores. El objetivo de este estudio fue identificar los factores de estrés, estresores y factores de riesgo en los conductores de autobuses urbanos en Aracaju, SE. Investigación caracterizada como diseño correlacional descriptivo de corte con el uso de los siguientes instrumentos de recolección:
Inventario de Síntomas de Estrés de Lipp (ISSL), Cuestionario Internacional de Actividad Física (IPAQ - 8,0) y la encuesta sociodemográfica. La muestra fue de 322 conductores seleccionados de manera representativa y voluntariamente dispuestos a participar. Todos los sujetos del estudio eran hombres (edad media de 37,16 \pm 8,79 años), el 62.11\% casados, el 27,02\% 
solteros, el 5,59\% divorciados, el 5,28\% tenía otra tipo de estado civil y el $81.99 \%$ tenía niños. Entre los entrevistados, el $46.89 \%$ de los sujetos tenían estrés y el $46.18 \%$ reportó el tipo de organización laboral como un estresor. De los que tenían algún grado de estrés, el 3,97\% se encontraba en la fase de alerta, el $88.74 \%$ en la fase de resistencia, el $1,99 \%$ en el corto agotamiento y el 5,29\% en el agotamiento. Los factores de riesgo relacionados con el estrés fueron: volumen de trabajo por encima de 8 horas/día; asaltos en el trabajo; estilo de vida sedentario; IMC superior a $25 \mathrm{~kg} / \mathrm{m}^{2}$ y menos de 6 horas de sueño/día. Los resultados también indican que el $77,02 \%$ de los sujetos de

\section{INTRODUÇ̃̃O}

Com o crescimento da urbanização, o transporte coletivo adquire uma importância social e econômica cada vez maior, conduzindo milhões de passageiros que deles dependem para satisfazer suas necessidades básicas. A atividade de dirigir estes veículos, no entanto, é desgastante, causa fadiga e sua eficácia está relacionada principalmente a fatores ambientais do local de trabalho e à forma como os motoristas desenvolvem estratégias de enfrentamento para lidar com estes fatores (BATTISTON; CRUZ; HOFFMANN, 2006).

$A$ atividade do motorista exige atenção constante, precisão na realização das ações, autocontrole, reflexo rápido (direção defensiva), análise e interpretação das ações fornecidas pelos equipamentos dos veículos, sendo considerada tarefa complexa principalmente por ser uma tarefa extra-muros da empresa, imprimindo maior possibilidades de imprevistos uma vez que envolve variáveis físicas, contato social, responsabilidade, desafio mental, carga de trabalho, trânsito, assaltos, bem como o desencadeamento de estresse ocupacional (MENDES, 1999). Essas situações podem influenciar em aspectos do trabalho que dizem respeito ao motorista, como a satisfação e a penosidade decorrente do processo de trabalho.

De um modo geral, o estresse é estudado por ter um efeito facilitador no desenvolvimento de muitas doen- investigación tienen bajos niveles de actividad física. Se observó una correlación negativa significativa entre el nivel de actividad física y el estrés ( $r=-0,238$, $p<0,001)$. La presencia de la fase de la resistencia al estrés sugiere que los conductores han desarrollado capacidad de recuperación a factores de estrés estudiados.

\section{PALABRAS CLAVE}

Agotamiento, Estilo de vida sedentario, Conductores de autobuses

ças, tais como hipertensão, infarto, artrite, asmas e problemas dermatológicos, especialmente se os agentes estressores foram constantes e não controlados. Isto acarreta em prejuízo para a qualidade de vida e a produtividade do homem, o que gera uma grande busca pelas causas e desenvolvimento de metodologias/ estratégias para sua redução (COSTA, 2006).

0 estresse é dividido em fases, sendo que na fase de alerta, o organismo sofre uma excitação de agressão ou de fuga ao estressor que pode ser entendido como comportamento de adaptação; na fase de resistência o estressor continua presente por longos períodos de tempo ou com intensidade maior e, o organismo faz uso de suas reservas de energia adaptativas, na tentativa de buscar o reequilíbrio interno; a fase de quase-exaustão é onde as defesas começam a ceder e o sujeito tem dificuldades de resistir às tensões e restabelecer 0 equilíbrio e, é na fase de exaustão que o estresse é considerado intenso e ultrapassa as reservas energéticas adaptativas, havendo uma quebra total da resistência, podendo aparecer exaustão psicológica nas formas de depressão e exaustão física, com o consequente aparecimento de doenças (NODARI et al., 2014).

Atualmente, a influência da atividade física na meIhoria da qualidade de vida e no controle do estresse tem sido muito divulgada. Os exercícios físicos redu- 
zem os níveis de ansiedade, depressão e raiva, considerados sintomas de estresse (NUNOMURA; TEIXEIRA; CARUSO, 2004), e também reduziriam a influência de fatores de estresse psicossociais sobre o indivíduo (REIS; FERNANDES; GOMES, 2010). Outro estudo que poderia corroborar a suposição de que o exercício físico regular ajudaria a reduzir os níveis de estresse é o de Steptoe (1994), no qual o autor relacionou uma série de estudos realizados nesse campo e revelou que, quanto melhor era o condicionamento físico dos indivíduos, mais favoráveis eram suas respostas ao estresse.

A atividade profissional, desenvolvida com o intuito de suprir o homem de suas necessidades básicas, tem se constituído de fator limitante da expectativa de vida dos indivíduos. Tal assertiva norteia o incentivo à realização de atividades físicas como promoção e prevenção da saúde no ambiente de trabalho (MONTEIRO et al., 1998). O corpo humano na sua relação com o ambiente, diante de algum evento importante que altere sua autorregulação orgânica, entra num processo de reequilíbrio, preparando-se para a ação, satisfazendo sua necessidade na busca pela homeostase. Esse processo de adaptação que consiste em uma série de modificações fisiológicas que requer esforço desgasta ou estressa o organismo, é denominado de estresse (SELYE, 1965).

Quando submetido à pressão psicológica ou a situações de risco real ou hipotético, o ser humano sofre uma superestimulação do sistema nervoso autônomo, havendo pouca liberação de acetilcolina, substância necessária a estimulação da resposta de descanso. A esse mecanismo denominou-se inicialmente de lutar ou fugir. Após vários trabalhos de definição desse mecanismo, Selye (1965) o definiu como um estado manifestado por uma síndrome específica, constituída por todas as alterações não-específicas produzidas num sistema biológico.

Nesse aspecto, observam-se dois tipos de alterações que o estresse causa: uma alteração primária, relacionada a uma lesão, que é induzida não especificamente em qualquer ponto do corpo por qualquer agente não específico; e uma alteração secundária, associada à defesa, que seria o desenvolvimento es- pecífico da síndrome de adaptação geral (SAG). Assim, o estresse significa uma agressão e, também uma defesa ao organismo (BATTISTON; CRUZ; HOFFMANN, 2006)

Tentar conhecer os fatores estressantes, sejam eles internos e/ou externos é uma das ações do indivíduo resiliente. Nota-se que, no caso dos motoristas de ônibus, a maioria dos eventos estressores vivenciados pelos mesmos não estão sob seu controle (congestionamento no trânsito, calor excessivo, excesso de passageiros, precariedade do veículo, assaltos). Dessa forma, torna-se relevante não só conhecer tais eventos propulsores de estresse, como também as características protetoras se desenvolveram e de que forma modificaram o percurso pessoal do indivíduo para que o mesmo se tornasse resiliente (PINHEIRO, 2004).

As estratégias de enfrentamento podem ter consequências positivas ou negativas, como por exemplo, um motorista que está ansioso no trânsito pode tanto fumar um cigarro como fazer um treino respiratório. Ambos atenuam a ansiedade, porém só o último está voltado para estratégias saudáveis, típicas na resiliência (ZANELATO, 2008).

Estudo de Rezende (2001) identificou aspectos da situação de trabalho e a presença de estresse ocupacional em motoristas de empresa de transporte coletivo urbano da cidade de Belo Horizonte-MG. Os dados obtidos indicaram que os motoristas possuem um bom nível de saúde física e de controle emocional, e, em decorrência, baixos níveis de estresse. Apesar disso, aponta que as situações de trabalho dos motoristas apresentam-se como fontes de pressão que estão diretamente correlacionadas com o estresse e que eles geralmente utilizam a racionalidade e o apoio social para enfrentar os problemas do seu cotidiano de trabalho.

Essa autora também menciona que motoristas que possuem mais tempo no exercício da profissão estão mais propensos a desenvolver quadros de estresse do que os novatos, o que pode indicar que, com o passar dos anos, a profissão de motorista de ônibus torna-se mais desgastante, causando danos à saúde, ou até mesmo o desejo de abandonar a empresa, mudar 
de profissão ou se aposentar. Este estudo tem como objetivo identificar estresse, estressores e fatores de riscos em motoristas de ônibus urbano de Aracaju-SE.

\section{MÉTODO}

A cidade de Aracaju, capital do Estado de Sergipe, tem seu sistema de transporte coletivo urbano regido por sete empresas de ônibus que se distribuem para o favorecimento da mobilidade dos indivíduos em uma área de $181,1 \mathrm{Km}^{2}$. A frota de ônibus municipal é composta por aproximadamente 530 veículos guiada por cerca de 1500 motoristas distribuídos nas respectivas empresas. O sistema viário municipal é composto de sete terminais de integração de ônibus, locais onde existe a permuta e repouso dos motoristas.

0 presente estudo se caracteriza por ser do tipo descritivo e transversal. A população, estimada em 1500 trabalhadores, segundo informações do Sindicato dos Trabalhadores de Transportes Rodoviários de Aracaju (SINTTRA), foi composta por motoristas de ônibus que perfazem linhas urbanas em Aracaju, sendo que a amostra foi definida e calculada pela fórmula proposta por Barbetta (2001). O quantitativo amostral do estudo consistiu de 322 motoristas de ônibus, sendo excluídos da amostra os motoristas com menos de três anos de serviço, afastados ou aposentados ou com alguma incapacidade clínica ou física. Também não fizeram parte da amostra aqueles que no dia da entrevista estavam ausentes ou que não aceitaram responder o instrumento de coleta de dados.

Os instrumentos utilizados para a coleta de informações foram: o Inventário de Sintomas de Estresse para Adultos de Lipp (ISSL), instrumento validado por Lipp (2001), que apresenta modelo quadrifásico do estresse: "Alerta”, "Resistência”, "Quase exaustão" e "Exaustão”. Visou identificar, objetivamente, se o motorista apresentava estresse e em que fase se encontrava. Foi utilizado também o questionário de levantamento sociodemográfico (informações biográficas, carga horária trabalhada, idade, estado civil, tempo de serviço e estressores ocupacionais) e o Questionário Internacional de Atividade Física - (IPAQ - 8.0, versão curta), que mensura o nível de atividade física e foi proposto pela Organização Mundial da Saúde (OMS) em 1998, validado em uma amostra da população brasileira, pelo Centro de Coordenação do IPAQ no Brasil - Centro de Estudos do Laboratório Aptidão Física de São Caetano do Sul (CELAFISCS) (MATSUDO et al., 2001).

Para análise dos dados foi utilizada a estatística descritiva para dados sociodemográficos, aplicado o Odds Ratio para estimar a razão de chance de os motoristas de ônibus desenvolverem estresse em relação à carga horária de trabalho, ao número de assaltos sofridos, ao nível de atividade física, ao índice de massa corporal e à quantidade de horas de sono. Foi utilizado ainda o teste de Mann Whitney para verificar os efeitos da carga horária de trabalho e o tempo de serviço no desenvolvimento de estresse nos motoristas, assim como o teste de comparação múltipla de médias de Duncan para aferir a diferença entre os níveis de estresse e o tempo de serviço na profissão. Foi calculado o coeficiente de correlação de Spearman para estimar a relação entre os níveis de estresse com os de atividade física, assim como os níveis de estresse com o número de assaltos sofridos. Os testes foram aplicados com uso do pacote estatístico SPSS 18.0, adotando-se o nível de confiabilidade de $95 \%$.

Todos os participantes da pesquisa foram esclarecidos, inicialmente pela leitura do Termo de Consentimento Livre e Esclarecido sobre objetivos e procedimentos da pesquisa. 0 projeto foi aprovado pelo Comitê de Ética e Pesquisa da Universidade Tiradentes sob o n 480508 . Os dados coletados foram utilizados exclusivamente para os fins previstos no protocolo. O Inventário de Sintomas de Estresse de Lipp (ISSL) (LIPP, 2001) foi adquirido por psicólogo registrado no Conselho Nacional de Psicologia e utilizado, respeitando os preceitos éticos do mesmo por se tratar de material com direitos autorais reservados. 


\section{RESULTADOS}

Todos os sujeitos do estudo são do sexo masculino com média de idade de $37,16 \pm 8,79$ anos, $62,11 \%$ são casados, $27,02 \%$ solteiros, $5,59 \%$ divorciados, $5,28 \%$ declararam ter outro tipo de situação conjugal e $81,99 \%$ dos sujeitos têm filhos, com média de 2,13 $\pm 1,05$ filhos. Foi constatado que $46,89 \%$ da amostra apresentou estresse, dos quais 3,97\% na fase de "alerta", 88,74\% na fase de "resistência", 1,98\% na “quase exaustão" e 5,29\% na fase de "exaustão".

Os principais estressores relatados foram a organização do trabalho, passageiros, trânsito e condições de ônibus (FIGURA 1). Após ajuste das variáveis no modelo de razão de chance, observou-se que os fatores que apresentam riscos para o estresse foram: carga horária acima de 8 horas/dia, o fato de ter sido assaltado, ausência de atividade física, IMC acima de $25 \mathrm{~kg} / \mathrm{m}^{2}$ ( $O R=1,69 ; p=0,036 ; \mathrm{IC}=1,06-2,68)$ e horas de sono menor que 6 horas/dia $(O R=1,47 ; p=0,136$; IC=0,92 - 2,34).

Figura 1 - Principais estressores relatados pelos motoristas de ônibus urbano da cidade de Aracaju-SE

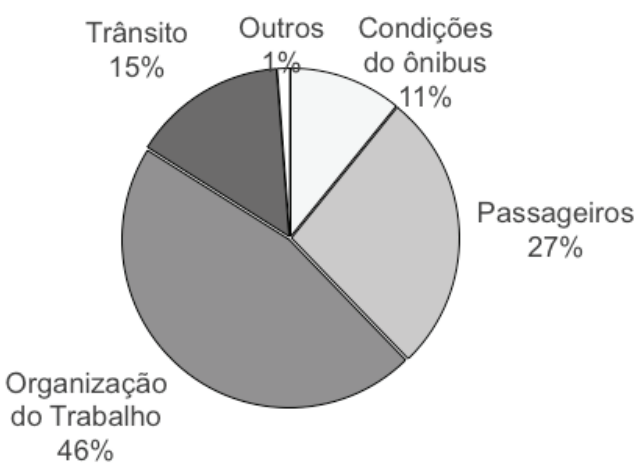

Fonte: Dados da pesquisa.

Constatou-se que $53,42 \%$ da amostra trabalha mais de 8 horas/dia (TABELA 1). Não foi encontrada diferença estatisticamente significativa entre os motoristas com e sem estresse e a carga horária trabalhada $(Z=1,95 ; p=0,061)$.
Foi encontrado nesse estudo, utilizando-se o Odds ratio, uma chance estimada de 1,45 vezes para aqueles com elevada carga horária de trabalho (acima de 8 horas por dia) no que se refere ao desencadeamento do estresse $(O R=1,45 ; p=0,126 ; I C=0,93-2,25)$.

Tabela 1 - Distribuição da relação entre carga horária trabalhada e ocorrência de estresse em motoristas de ônibus em Aracaju, SE

\begin{tabular}{llll}
\multicolumn{4}{c}{ Carga Horária Diária } \\
\hline Estresse & $\begin{array}{l}\text { Até } 8 \\
\text { horas/dia }\end{array}$ & $\begin{array}{l}\text { Acima de } 8 \\
\text { horas/dia }\end{array}$ & Total \\
\hline $\begin{array}{l}\text { Com } \\
\text { estresse }\end{array}$ & $19,57 \%$ & $27,32 \%$ & $46,89 \%$ \\
$\begin{array}{l}\text { Sem } \\
\text { estresse }\end{array}$ & $27,02 \%$ & $26,09 \%$ & $53,11 \%$ \\
Total & $46,58 \%$ & $53,42 \%$ & $100,00 \%$ \\
\hline
\end{tabular}

Fonte: Dados da pesquisa.

Não foi encontrada diferença significativa entre as médias de tempo de serviço e a presença de estresse entre os motoristas de ônibus ( $Z=0,43 ; p=0,663)$. Esse resultado demonstra que não existe uma tendência ao desencadeamento do estresse com o passar dos anos para esse grupo profissional.

Tabela 2 - Média de tempo de serviço prestado (em anos) e as fases do estresse em motoristas de ônibus, Aracaju, SE

\begin{tabular}{|c|c|c|c|c|c|c|}
\hline & & \multicolumn{5}{|c|}{ Fases do Estresse } \\
\hline & & $\begin{array}{l}\text { Sem } \\
\text { Estresse }\end{array}$ & Alerta & Resistência & $\begin{array}{l}\text { Quase } \\
\text { Exaustão }\end{array}$ & Exaustão \\
\hline Tempo & Média & $10,29 a$ & $12,33 a$ & $10,20 a$ & $15,67 a$ & $7,87 a$ \\
\hline $\begin{array}{l}\text { de } \\
\text { Seniço }\end{array}$ & $\begin{array}{l}\text { Desvio } \\
\text { Padrão }\end{array}$ & $\pm 7,46$ & $\pm 8,28$ & $\pm 7,16$ & $\pm 4,00$ & $\pm 3,09$ \\
\hline
\end{tabular}

Médias com a mesma letra não são estatisticamente diferentes

Fonte: Dados da pesquisa. 
Foi encontrada correlação negativa e significativa entre o nível de atividade física e o de estresse na amostra estudada ( $r s=-0,24 ; p<0,001$ ), indicando que quanto menor o nível de atividade física, maior a propensão dos motoristas de ônibus na fase de estresse. Quando utilizada a razão de chance com ponto de corte "ter ou não estresse" e possuir ou não algum tipo de atividade física ("sem atividade", "ativo" ou "muito ativo"), observa-se que há risco estimado de 2,29 vezes de o sujeito sem atividade física desenvolver o estresse $(O R=2,29 ; p<0,001$; $\mathrm{IC}=1,47-3,59$ ).

A prevalência de motoristas de ônibus aracajuanos que apresentam níveis de atividade física diárias abaixo do recomendável, ou seja, na faixa de risco à saúde, foi de $77,01 \%$.

Figura 2 - Nível de atividade física dos motoristas de ônibus urbano da cidade de Aracaju-SE

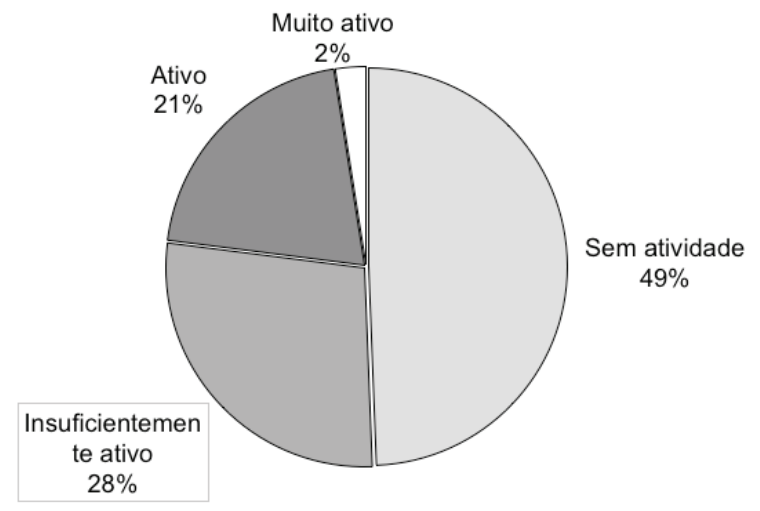

Fonte: Dados da pesquisa.

Dos sujeitos investigados, $62,42 \%$ sofreram assaltos. Esse tipo de violência aumenta em 1,59 vezes a chance de o motorista desenvolver estresse ( $R C=1,59 ; p=0,057 ; I C=1,01-2,53)$, além de apresentar uma correlação positiva e significativa entre a quantidade de assaltos sofridos e o nível de estresse apresentado ( $r s=0,151 ; p=0,007$ ), ou seja, quanto maior o número de assaltos, mais avançada é a fase do estresse.

\section{DISCUSSÃO}

Os motoristas de ônibus da capital Sergipana apresentam um nível de estresse abaixo da média, principalmente comparando com outros estudos, como o de Nascimento e Pasqualetto (2002), que aponta $80 \%$ dos motoristas em geral com estresse em Goiânia, GO; e o estudo de Matos, Moraes e Pereira (2015) com estresse em $54,8 \%$ dos motoristas em Belo Horizonte-MG. Apesar de grande parte dos motoristas apresentarem estresse, não se pode generalizar que esta seja uma profissão estressante, pois, o estresse pode ser decorrente de uma variedade de fatores, alguns relacionados às condições ambientais e laborais, ou das próprias características individuais (LIPP; MALAGRIS, 1995).

Por outro lado, observa-se que os motoristas de ônibus têm propensão a sofrer de alta carga mental, em virtude do excesso de horas trabalhadas e da pressão para cumprir o trajeto em tempo, dos períodos de pausas insuficientes entre as viagens, faltando tempo inclusive para as necessidades fisiológicas (ONOZATO; RAMOS, 2001).

Segundo Almeida (2002), em estudo realizado com motoristas profissionais observou-se que o estresse teve uma maior incidência entre os motoristas de ônibus e estava relacionada também com a rotina de itinerários, as constantes paradas, a conferência de horário e o tumulto de ônibus lotados. Um dos estressores laborais mais apontados pelos sujeitos estudados em Aracaju-Se foi à quantidade de assaltos a transportes coletivos. A violência e os assaltos no interior dos ônibus de Aracaju são problemáticas bastante discutidas entre os motoristas. Por outro lado, segundo dados da Coordenadoria de Estatística e Análise Criminal (CEACRIM), órgão da Secretaria de Segurança Pública-SE, apresentou o número de roubos aos veículos de transporte coletivos em Aracaju e região metropolitana, totalizando 1207 em 2008, apresenta tendência de redução, com 714 casos em 2009 e 417 em 2010 e uma redução de $25 \%$ no primeiro semestre 2011 , em relação ao mesmo período de 2010. 
A prática de atividade física parece sugerir uma estratégia para o controle do estresse ao promover resposta de descanso, uma vez que diante de situações de risco, pressões psicológicas e ameaças reais/ imaginárias, o sistema nervoso autônomo sofre uma super-estimulação, necessária para a mobilização de uma ação que, quando impedido de agir, a resposta de descanso não vem ou é dificultada, sendo a atividade física um meio de liberação de acetilcolina (FERMINO; PEZZINI; REIS, 2010).

O sedentarismo observado entre os motoristas de ônibus urbanos de Aracaju, com proporção similar a observada por Deus (2005), em motoristas de ônibus de Florianópolis-SC, é um fator de risco, pois a atividade física, incluída nos hábitos saudáveis, pode ser utilizada no combate e prevenção de agravos à saúde (ACSM, 1998; PALMA, 2000; NAHAS, 2006).

Em estudo realizado por Barros e Santos (2000) acerca dos comportamentos de risco em trabalhadores da indústria, foi detectado que $42,2 \%$ dos sujeitos não realizavam atividades físicas e destes, 13,9\% referiram níveis elevados de estresse e dificuldade para enfrentar a vida. Ainda nesse estudo foi encontrado que a percepção de estresse entre os homens foi inversamente associada à prática de atividades físicas de lazer.

\section{CONCLUSÃO}

Um percentual importante dos motoristas de ônibus urbanos em Aracaju apresenta quadro de estresse e esse evento está relacionado com a carga horária trabalhada e com a violência (assalto no interior do coletivo) sofrida pelos sujeitos. Dos sujeitos estressados, a maioria deles se encontrava na fase de resistência. A predominância desta fase do estresse pode ser um indicativo de que os fatores estressores continuam presentes e o organismo tenta restabelecer o equilíbrio.

A elevada taxa de sujeitos na faixa de níveis abaixo dos recomendáveis de atividades físicas, bem como o percentual elevado de sujeitos com estresse, torna-se um agravante na saúde do trabalhador motorista de ônibus urbano, pois, além dos riscos relacionados ao sedentarismo, como o desencadeamento de doenças cardiovasculares, o estresse pode ser um dos maiores fatores de risco para a saúde e qualidade de vida dos trabalhadores.

Foi encontrada uma correlação negativa e significativa entre o nível de atividade física e os níveis de estresse, com risco aproximado duas vezes maior em desenvolver estresse pela falta de qualquer tipo de atividades físicas recomendáveis. Além disso, tem-se que o fato do estressor "organização do trabalho" ser relatado por grande parte dos motoristas como principal causa do estresse, estratégias organizacionais que poderiam fazer parte do programa semanal ou mensal dos trabalhadores, principalmente no que se refere a jornadas de trabalho, adequação da carga horária e até mesmo na estrutura dos locais para repousos e permutas dos motoristas, com espaço adequado para lazer e atividade física para esses trabalhadores.

Faz-se necessário que as autoridades de trânsito busquem, de forma integrada com a sociedade, encontrar soluções para a prevenção dos graves problemas de circulação de veículos e violência no trânsito, uma vez que este é considerado um fator importante e contribuinte para o estresse. Concomitantemente a isto, cabe aos empresários de transportes urbanos buscar a melhoria das condições e qualidade laborais de seus funcionários.

\section{REFERÊNCIAS}

ACSM (American College of Sports Medicine). The recommended quantity and quality of exercise for developing and maintaining cardiorespiratory and fitness, and flexibility in healthy adults. Med. Sci. Sports Exerc., Madison, v.30, n.6, p.975-91, 1998.

ALMEIDA, N.D.V. Contemporaneidade X Trânsito: Reflexão psicossocial do trabalho dos motoristas de coletivo urbano. Psicol. cienc. prof., Brasília, v.22, n.3, p.62-69, 2002.

\section{BARBETTA, P.A. Estatística aplicada às ciências}

sociais. Florianópolis: UFSC, 2001. 
BARROS, M.V.; SANTOS, S.G.A. Atividade física como fator de qualidade de vida e saúde do trabalhador. Rev. Virtual Ergon. UFSC, Florianópolis, 2000. Disponível em: <http://portalbiocursos.com.br/ohs/ data/docs/51/110-_A_ATIVIDADE_FYSICA_COMO FATOR_DE_QUALIDADE_DE_VIDA_E_SAYDE_DO_ TRABALHADOR.pdf>. Acesso em: 25 ago. 2014.

BATTISTON, M.; CRUZ, R.M.; HOFFMANN, M.H. Condições de trabalho e saúde de motoristas de transporte coletivo urbano. Estud psicol., Campinas, n.11, v.3, p.333-343, 2006.

COSTA, E.A.V.G. Estudos dos constrangimentos físicos e mentais sofrido pelos motoristas de ônibus urbano da cidade do Rio de Janeiro. 2006. 154f. Dissertação (Mestrado) - Universidade Católica do Rio de Janeiro, Rio de Janeiro, 2006.

DEUS, M.J. Comportamentos de risco à saúde e estilo de vida em motoristas de ônibus urbanos: recomendações para um programa de promoção de saúde. 2005. 175f. Tese (Doutorado) - Universidade Federal de Santa Catarina, Florianópolis, 2005.

FERMINO, R.C.; PEZZINI, M.R.; REIS, R.S. Motivos para prática de atividades física e imagem corporal em frequentadores de academia. Rev. Bras. Med. Esporte, São Paulo, v.16, n.1, p.18-23, 2010.

\section{LIPP, M.E.N. Inventário de sintoma de stress de} Lipp. São Paulo: Casa do Psicólogo, 2001.

LIPP, M.E.N.; MALAGRIS, L.E.N. Manejo do Estresse. In: RANGE, B. Psicoterapia comportamental e cognitiva: pesquisa, prática, aplicações e problemas. Campinas: Psy II:. 1995. p.279-292.

MATOS, M.G.; MORAES, L.F.R.; PEREIRA, L.Z. Análise do estresse ocupacional em motoristas de coletivo urbano na cidade de Belo Horizonte. Rev. Gest. Tecnol.; Pedro Leopoldo, v.15, n.1, p.256-275, 2015.
MATSUDO, S. et al. Questionário Internacional de Atividade Física (IPAQ): estudo de validade e reprodutibilidade no Brasil. Rev. Bras. Ativ. Fis. e Saúde, Londrina, v. 6, n.2, p.5-18, 2001.

MENDES, L.R. Condições de trabalho no transporte coletivo: desgaste e responsabilidade do motorista de ônibus. In: SAMPAIO, J.R. (Org.). Qualidade de vida, saúde mental e psicologia social. São Paulo: Casa do psicólogo, 1999. p.153-180.

MONTEIRO, H.L. et al. Fatores socioeconômicos e ocupacionais e a prática de atividade física regular: estudo a partir de policiais militares em Bauru, SP. Motriz: Rev. Educ. Fis., Rio Claro, v.4, n.2, p.91-97, 1998.

NAHAS, M.V. Atividade física, saúde e qualidade de vida: conceitos e sugestões para um estilo de vida ativo. Londrina: Midiograf, 2006.

NASCIMENTO, L.P.; PASQUALETTO, A. Estresse como agente propulsor do aumento dos acidentes de trânsito em Goiânia. Estudos: Rev. UCG. [on-line] 2002. Disponível em: <http://www2.ucg.br/nupenge/ pdf/artigo_06.pdf>. Acesso em: 25 ago. 2014.

NODARI, N.L. et al. Estresse, conceitos, manifestações e avaliação em saúde: revisão de literatura. Rev. Saúde e Desenvolv. Humano, Canoas, v.2, n.1, p.61-74, 2014.

NUNOMURA, M.; TEIXEIRA, L.A.C.; CARUSO, M.R.F. Nível de estresse em adultos após 12 meses de prática regular de atividade física. REMEFE, São Paulo, v.3, n.3, p.125-134, 2004.

ONOZATO, E.; RAMOS, S.P. O estresse na profissão de motorista do transporte coletivo urbano por ônibus. I Jornada Científica do Centro-Oeste de Economia e Administração, Anais... Campo Grande. CD-ROM, 2001. 
PALMA, A. Atividade física, processo saúde-doença e condições sócio-economicas: uma revisão de literatura. Rev. Paulista Educ. Fís., São Paulo, v.14, n.1, 2000.

PINHEIRO, D.P.N. A resiliência em discussão. Psicol. estud., Maringá, v.9, n.1, p.67-75, 2004.

REIS, A.L.P.P.; FERNANDES, S.R.P.; GOMES, A.F. Estresse e fatores psicossociais. Psicol. cienc. prof., Brasília, v.30, n.4, p.712-725, 2010.

REZENDE, A.L.M. Situações de trabalho e riscos ocupacionais: um estudo de caso com os motoristas de transporte urbano por ônibus. 2001. $93 f$. Dissertação (Mestrado) - Universidade Federal de Minas Gerais, Belo Horizonte, 2001.

SELYE, H.A. Tensão da vida. São Paulo: Ibrasa, 1965.

STEPTOE, A. Aerobic exercise, stress and health. Proceedings of the VIII European Congress of Sport Psychology 1991. Koeln, 1994.

\section{ZANELATO, L.S. Manejo do stress, coping e} resiliência em motoristas de ônibus urbano.

2008. 116f. Dissertação (Mestrado) - Universidade Estadual Paulista, Bauru, 2008. 